\title{
Kinetics of Exocytosis Is Faster in Cones Than in Rods
}

\author{
Katalin Rabl, ${ }^{1}$ Lucia Cadetti, ${ }^{1}$ and Wallace B. Thoreson ${ }^{1,2}$ \\ Departments of ${ }^{1}$ Ophthalmology and Visual Sciences and ${ }^{2}$ Pharmacology, University of Nebraska Medical Center, Omaha, Nebraska 68198-5840
}

Cone-driven responses of second-order retinal neurons are considerably faster than rod-driven responses. We examined whether differences in the kinetics of synaptic transmitter release from rods and cones may contribute to differences in postsynaptic response kinetics. Exocytosis from rods and cones was triggered by membrane depolarization and monitored in two ways: (1) by measuring EPSCs evoked in second-order neurons by depolarizing steps applied to presynaptic rods or cones during simultaneous paired whole-cell recordings or (2) by direct measurements of exocytotic increases in membrane capacitance. The kinetics of release was assessed by varying the length of the depolarizing test step. Both measures of release revealed two kinetic components to the increase in exocytosis as a function of the duration of a step depolarization. In addition to slow sustained components in both cell types, the initial fast component of exocytosis had a time constant of $<5 \mathrm{~ms}$ in cones, $>10$-fold faster than that of rods. Rod/cone differences in the kinetics of release were substantiated by a linear correlation between depolarization-evoked capacitance increases and EPSC charge transfer. Experiments on isolated rods indicate that the slower kinetics of exocytosis from rods was not a result of rod-rod coupling. The initial rapid release of vesicles from cones can shape the postsynaptic response and may contribute to the faster responses of cone-driven cells observed at light offset.

Key words: ribbon synapse; capacitance measurement; retina; tiger salamander; photoreceptor; postsynaptic current

\section{Introduction}

In the vertebrate retina, rod and cone photoreceptors are relatively depolarized in darkness with membrane potentials of -35 to $-45 \mathrm{mV}$, allowing tonic release of the neurotransmitter L-glutamate through a continuous cycle of exocytosis and endocytosis. Light-evoked hyperpolarizing voltage responses of photoreceptors are graded in amplitude with intensity. To vary transmitter release smoothly and continuously with changes in membrane potential, photoreceptors use L-type calcium channels that exhibit sustained activation (Corey et al., 1984; Wilkinson and Barnes, 1996; Schmitz and Witkovsky, 1997; Thoreson et al., 1997).

Cone-driven light responses of second-order retinal neurons (bipolar and horizontal cells) are considerably faster than roddriven light responses (Witkovsky and Stone, 1983). The light responses of cones are likewise 5-10 times faster than rod light responses (Baylor and Hodgkin, 1973; Pasino and Marchiafava, 1976). The faster kinetics of cone light responses compared with rod responses is primarily attributable to differences in outersegment $\mathrm{Ca}^{2+}$ homeostasis and the inactivation of cascade enzymes by $\mathrm{Ca}^{2+}$ (for review, see Korenbrot and Rebrik, 2002). However, the faster postsynaptic responses of cone-driven neurons are not simply a consequence of faster cone light responses but also involve faster synaptic transmission in the cone pathway. By simultaneously recording from photoreceptors and ganglion

Received 0ct. 20, 2004; revised April 4, 2005; accepted April 5, 2005.

This work was supported by the National Eye Institute (EY-10542), Gifford Foundation, Lion's Clubs, Fight for Sight, and Research to Prevent Blindness. We thank Eric Bryson for his technical assistance and Ruth Heidelberger for her comments on this manuscript.

Correspondence should be addressed to Wallace B. Thoreson, Department of Ophthalmology, University of Nebraska Medical Center, Durham Research Center, Omaha, NE 68198-5840. E-mail: WBTHORES@UNMC.EDU.

DOI:10.1523/JNEUROSCI.4298-04.2005

Copyright $\odot 2005$ Society for Neuroscience $\quad$ 0270-6474/05/254633-08\$15.00/0 cells, Baylor and Fettiplace (1977) found that responses of ganglion cells to depolarizing current injected into cones were about threefold faster than rod-driven responses. And, by comparing simultaneously recorded light responses in photoreceptors and horizontal cells from the turtle eyecup, Copenhagen and colleagues (Schnapf and Copenhagen, 1982; Copenhagen et al., 1983) concluded that the impulse responses of rod and cone synapses were similar in shape but 8-10 times faster for cone synaptic output.

In the present study, we directly examined the kinetics of exocytosis from rods and cones in the retinal slice preparation, in which synaptic terminals and synaptic contacts remain mostly intact. Two complementary techniques were used to assess release: capacitance measurement techniques and recordings of the postsynaptic current (PSC) evoked by presynaptic stimulation during simultaneous paired recordings. The kinetics of release was assessed by varying the duration of a depolarizing pulse used to evoke release. Both methods revealed the presence of two kinetic components to release from rods and cones, suggesting the presence of two distinct pools of releasable vesicles. Furthermore, the fast component of release from cones was $>10$-fold faster than that of rods. Although other factors are also important, the rapid kinetics of release from cones may contribute to rapid conedriven responses in bipolar and horizontal cells.

\section{Materials and Methods}

Preparations. Experiments were performed using retinas from the aquatic tiger salamander (Ambystoma tigrinum; $18-25 \mathrm{~cm}$ ). Care and handling procedures were approved by the University of Nebraska Medical Center Institutional Animal Care and Use Committee. Animals were killed by decapitation, the eyes were enucleated, and the anterior portion of the eyes including the lens was removed. For preparation of retinal slices, the resulting eyecup was cut into quarters, and a section of eyecup was placed vitreal side down on a piece of filter paper $(2 \times 5 \mathrm{~mm}$; Type 
AAWP; $0.8 \mu \mathrm{m}$ pores; Millipore, Bedford, MA). After adhering to the filter paper, the retina was isolated under cold amphibian superfusate. Retinal slices $(125 \mu \mathrm{m})$ were cut with a razor-blade tissue chopper (Stoelting, Wood Dale, IL) and positioned in the recording chamber to allow viewing of the retinal layers with an upright fixed-stage microscope [BHWI (Olympus, Tokyo, Japan) or E600 FN (Nikon, Tokyo, Japan)].

For EPSC recordings, slices were superfused at $\sim 1 \mathrm{ml} / \mathrm{min}$ with an oxygenated solution containing the following (in $\mathrm{mm}$ ): $111 \mathrm{NaCl}, 2.5$ $\mathrm{KCl}, 1.8 \mathrm{CaCl}_{2}, 0.5 \mathrm{MgCl}_{2}, 10$ HEPES, 5 glucose, 0.1 picrotoxin, and 0.001 strychnine, $\mathrm{pH}$ 7.8. For recordings of capacitance changes, the superfusate contained the following (in $\mathrm{mm}$ ): $95 \mathrm{NaCl}, 2.5 \mathrm{KCl}, 2 \mathrm{CaCl}_{2}$, $0.5 \mathrm{MgCl}_{2}, 5 \mathrm{CsCl}, 10$ tetraethylammonium chloride (TEACl), 10 HEPES, 5 glucose, and 0.2 niflumic acid, $\mathrm{pH}$ 7.8. Niflumic acid, TEACl, and $\mathrm{CsCl}$ were included to minimize voltage and $\mathrm{Ca}^{2+}$-activated conductances. The presence of HEPES minimized effects of vesicular protons in the synaptic cleft (DeVries, 2001). Osmolarity was tested with a vapor pressure osmometer (Wescor, Logan, UT) and adjusted, if necessary, to $\sim 242 \mathrm{mOsm}$. Unless otherwise specified, chemicals were obtained from Sigma (St. Louis, MO).

Isolated rods were enzymatically dissociated with papain as described by Thoreson et al. (2004) and plated on glass slides coated with concanavalin A $(1 \mathrm{mg} / \mathrm{ml})$. Niflumic acid was omitted during recordings from isolated cells because it inhibited calcium currents $\left(I_{\mathrm{Ca}}\right)$ (Thoreson et al., 2003) and thus often blocked capacitance increases evoked by short depolarizing steps. As described in Results, tail currents evoked by long depolarizing steps in the absence of niflumic acid did not appear to significantly influence capacitance measurements, because the amplitude and time course of tail currents did not correlate with capacitance changes, and tests with a model cell circuit showed that large changes in membrane resistance simulating large tail currents produced no detectable changes in capacitance.

Electrophysiology. Ruptured-patch whole-cell recordings were obtained using 8-15 $\mathrm{M} \Omega$ patch electrodes pulled from borosilicate glass (outer diameter, $1.2 \mathrm{~mm}$; inner diameter, $0.95 \mathrm{~mm}$; with internal filament; World Precision Instruments, Sarasota, FL) on a PP-830 micropipette puller (Narishige USA, East Meadow, NY). The pipette solution contained the following (in $\mathrm{mm}$ ): $48 \mathrm{Cs}$ gluconate, $42 \mathrm{CsCl}$, $9.4 \mathrm{TEACl}$, $1.9 \mathrm{MgCl}_{2}, 9.4 \mathrm{MgATP}, 0.5 \mathrm{GTP}, 0.5 \mathrm{EGTA}$, and 32.9 HEPES, pH 7.2. The osmolarity was adjusted, if necessary, to $\sim 242 \mathrm{mOsm}$. For capacitance recording, pipettes were coated with Sylgard (Dow Corning, Midland, MI) to reduce stray capacitance. Residual pipette capacitance was compensated electronically. Superfusate was removed by suction to maintain a constant bath volume and thereby prevent changes in stray pipette capacitance.

Capacitance measurements were made using the "track-in" mode of the Optopatch patch-clamp amplifier, in which real and imaginary outputs from a lock-in amplifier provide negative feedback to the resistance and capacitance control settings, and a $70 \mathrm{~Hz}$ sine wave is applied to continuously optimize the phase setting. A detailed description of capacitance measurements using this amplifier is provided by Johnson et al. (2002). The holding potential was varied sinusoidally $(500-600 \mathrm{~Hz} ; 30$ $\mathrm{mV}$ peak to peak) about a mean holding potential of $-70 \mathrm{mV}$. Track-in feedback gain was increased to its highest stable value (typically $500-$ 1000). The amplifier output of membrane current, membrane capacitance, and series resistance were acquired and analyzed with a Digidata 1200 interface and pClamp 8.1 software (Axon Instruments, Foster City, CA). (Membrane conductance output is not explicitly provided by the Optopatch phase-lock amplifier.) Currents were low-pass filtered at 2 $\mathrm{kHz}$ and capacitance changes at $100 \mathrm{~Hz}$. Cells were stimulated by depolarizing steps to $-10 \mathrm{mV}$ from the mean holding potential of $-70 \mathrm{mV}$. Lock-in signals were gated out during the step and for $3 \mathrm{~ms}$ afterward to avoid effects of gating charges on capacitance measurements. Capacitance changes were measured $25-50 \mathrm{~ms}$ after the step to avoid overshoots than can sometimes be produced by the positive feedback phase tracking circuitry during the first $25 \mathrm{~ms}$ after the step (Johnson et al., 2002). As discussed further in Results, there was minimal endocytosis during the first $25 \mathrm{~ms}$.

In a small number of experiments, we measured capacitance using a dual sine wave protocol implemented using jClamp software (Scisoft,
New Haven, CT). In these experiments, a voltage stimulus ( $15 \mathrm{mV}$ peak to peak) of 390.6 and $781.2 \mathrm{~Hz}$ was applied about the DC holding potential, and admittance analysis was performed on the fast Fourier transform of the current response every $5.12 \mathrm{~ms}$ to provide estimates of membrane capacitance, series resistance, membrane resistance, and membrane current.

With both approaches, an identical step applied after $\sim 30$ s typically produced the same capacitance response, allowing multiple measurements from each cell. The presentation sequence of stimuli was varied between cells to avoid possible order effects. The response evoked by a $100 \mathrm{~ms}$ depolarizing step $(-70$ to $-10 \mathrm{mV}$ ) was retested periodically to check for response rundown.

Rods and cones were distinguished by the position and shape of their cell bodies as well as their characteristic outer segments, when present (Sherry et al., 1998; Stella and Thoreson, 2000). Passive membrane properties and access resistance were assessed using the membrane test function in Clampex (pClamp 8.1; Axon Instruments). Recordings in which the access resistance was $>50 \mathrm{M} \Omega$ were rejected; access resistance among remaining cells averaged $36.0 \pm 1.3 \mathrm{M} \Omega(n=51)$. Preliminary experiments revealed no obvious differences in capacitance responses from cells with or without outer segments aside from a greater capacitance noise among cells with larger whole-cell capacitance. We conducted most of our experiments on cells with outer segments, because they seemed to be healthier than cells lacking outer segments. The capacitance of rods and cones with outer segments averaged $22.6 \pm 1.1 \mathrm{pF}(n=29)$ and $46.5 \pm 3.2 \mathrm{pF}(n=28)$, respectively. Rods and cones without outer segments exhibited whole capacitance values of $11.5 \pm 0.5 \mathrm{pF}(n=19)$ and $18.0 \pm 1.8 \mathrm{pF}(n=7)$. Capacitance transients evoked by depolarizing steps in cells with outer segments were typically well fit by singleexponential functions with time constants averaging $2.1 \pm 0.1$ and $2.8 \pm$ $0.2 \mathrm{~ms}$ in rods $(n=29)$ and cones $(n=33)$, respectively. The finding that rods and cones appear to be isopotential is consistent with measurements made by Lasater et al. (1989), in which they recorded simultaneously from the terminal and inner segment of turtle cones. Input resistance $\left(R_{\text {in }}\right)$ of rods and cones with acceptable holding currents $(<200 \mathrm{pA}$ at $-70 \mathrm{mV}$ ) averaged $1.1 \pm 0.1 \mathrm{G} \Omega$ and $960 \pm 140 \mathrm{M} \Omega$, respectively. Consistent with the finding of Taylor and Morgans (1998) that cone input resistance was not correlated with total cell capacitance, there was not a statistically significant difference in the input resistance of photoreceptor cells with or without segments.

For paired recordings, rods or cones were voltage clamped simultaneously with adjacent postsynaptic neurons using a Multiclamp patchclamp amplifier (Axon Instruments). Both recording pipettes were positioned with Huxley-Wall micromanipulators (Sutter Instruments, Novato, CA). Currents were acquired using a Digidata 1322 interface and pClamp 8.1 software (Axon Instruments). Photoreceptors were voltage clamped at $-70 \mathrm{mV}$, bipolar cells at $-50 \mathrm{mV}$, and horizontal cells at -40 $\mathrm{mV}$. Horizontal cells exhibit outward light-evoked currents, inward currents evoked by depolarizing steps applied to presynaptic rods, and large inwardly rectifying voltage-dependent currents. OFF bipolar cells also show outward light-evoked currents and inward currents evoked by presynaptic depolarization but typically exhibit a higher input resistance and a current-voltage profile dominated by outwardly rectifying currents. ON bipolar cells show an outwardly rectifying current-voltage profile similar to OFF bipolar cells but inward light-evoked currents. The identities of presynaptic and postsynaptic cells were confirmed anatomically by adding a fluorescent tracer, Lucifer yellow $(2 \mathrm{mg} / \mathrm{ml})$, to the pipette solution. Charging curves for bipolar cells are well fit by single exponentials, indicating a compact electrotonic structure (Thoreson and Miller, 1996; Thoreson et al., 2004).

The criterion for statistical significance was chosen to be $p<0.05$ and evaluated with $F$ tests for comparisons of different curve fits or Student's $t$ tests for comparisons of different samples using GraphPad Software (San Diego, CA) Prism 4. Variability is reported as \pm SEM.

\section{Results}

To compare synaptic transmission from rods and cones, we recorded simultaneously from photoreceptors and second-order retinal neurons using a retinal slice preparation. Glutamate re- 


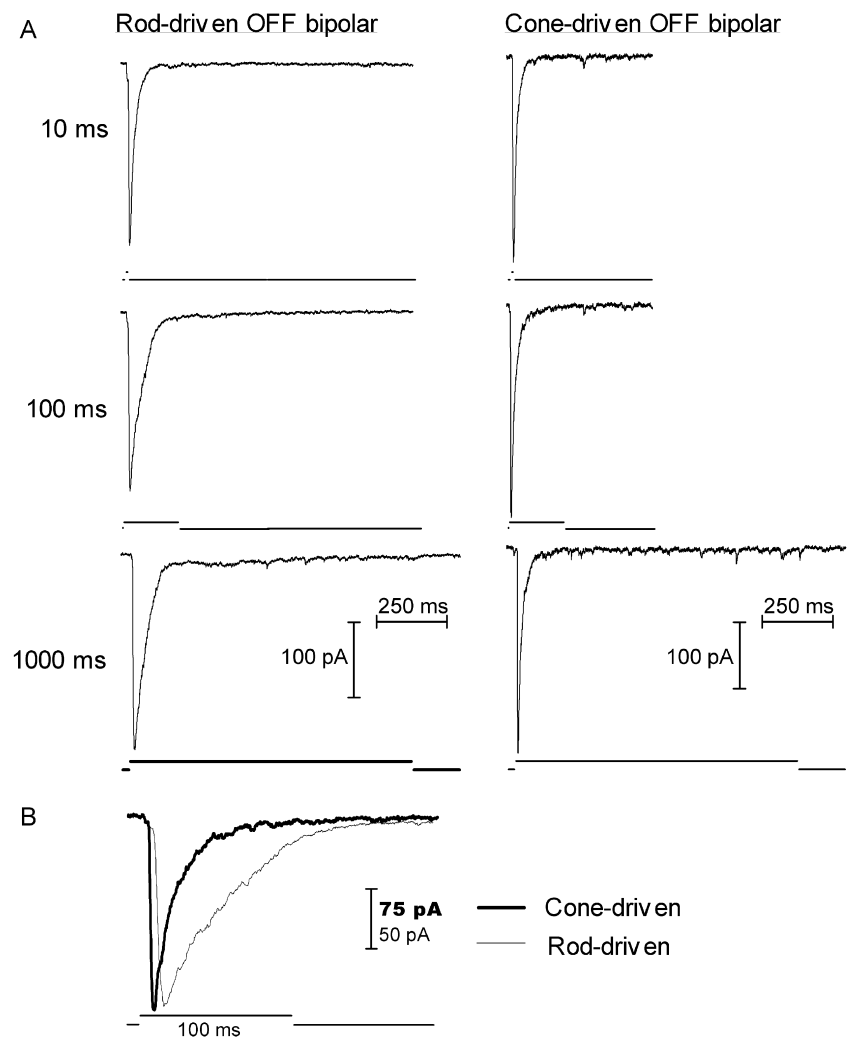

Figure 1. Depolarizing steps $(-70$ to $-10 \mathrm{mV})$ applied to rods or cones evoked transient EPSC in simultaneously recorded OFF bipolar cells. Cyclothiazide $(0.1 \mathrm{~mm})$ was added to inhibit AMPA receptor desensitization. $\boldsymbol{A}$, EPSCs evoked by steps of 10,100 , and 1000 ms duration. $\boldsymbol{B}$, Overlay of EPSCs evoked by $100 \mathrm{~ms}$ steps in rod-driven (thin trace) and cone-driven (thick trace) OFF bipolar cells shows that cone-driven EPSCs exhibited faster onset and offset than rod-driven EPSCS.

lease from photoreceptor terminals was stimulated with depolarizing steps from -70 to $-10 \mathrm{mV}$ applied to rods or cones. To analyze the kinetics of release, we varied the duration of the presynaptic depolarization (rods, 10-1000 ms; cones, 10-2000 ms) while simultaneously recording the EPSCs from synaptically coupled OFF bipolar or horizontal cells. Cyclothiazide $(0.1 \mathrm{~mm})$ was added to block AMPA receptor desensitization. Consistent with previous results indicating that glutamate receptors in these cells are primarily AMPA-type receptors (Maple et al., 1999), cyclothiazide made EPSCs less transient in every horizontal or OFF bipolar cell used for this study.

Figure 1 shows EPSCs evoked in an OFF bipolar cell by depolarization of a presynaptic rod (left column) and EPSCs in a different OFF bipolar cell stimulated by a presynaptic cone (right column). Increasing the step duration beyond $10 \mathrm{~ms}$ did not noticeably increase the amplitude of the EPSC in either the conedriven or rod-driven OFF bipolar cell. The most prominent change in the EPSCs with increasing step duration was a slight broadening of the EPSC in the rod-driven OFF bipolar cell when the step duration was increased from 10 to $100 \mathrm{~ms}$ (Fig. 1A). This broadening of the EPSC waveform is consistent with release of a larger number of vesicles by a $100 \mathrm{~ms}$ step than a $10 \mathrm{~ms}$ step in rods. The smaller change in the cone-driven EPSC observed under the same conditions suggests that there was a smaller increase in the number of vesicles released by the cone. In both the rodand cone-driven cells, the EPSCs subsided within $100 \mathrm{~ms}$, even when the step duration was increased to $1000 \mathrm{~ms}$. The finding that the EPSC decayed to near baseline in the presence of cy- clothiazide suggests that the transient nature of the initial component is not caused by glutamate receptor desensitization. The decay in the EPSC may instead reflect the depletion of releasable vesicles after an initial burst of exocytosis.

EPSCs evoked in cone-driven cells were more transient than those in rod-driven cells, even in the presence of cyclothiazide. This difference can be seen in Figure $1 B$ by overlaying EPSCs from rod- and cone-driven OFF bipolar cells. Both the initial rise and subsequent decay of the EPSC were faster in the cone-driven OFF bipolar cell (thick trace) than the rod-driven OFF bipolar cell (thin trace). To quantify differences in EPSC kinetics among rod- and cone-driven cells, the initial increase and subsequent decay of EPSCs obtained in cyclothiazide were each fit with single-exponential functions. Responses were included in this analysis if the time course could be adequately fit with a single exponential. In cone-driven OFF bipolar and horizontal cells, EPSCs rose and fell with time constants that averaged $7.0 \pm 2.0$ $\mathrm{ms}(n=8)$ and $19.1 \pm 2.6 \mathrm{~ms}(n=8)$, respectively. In rod-driven OFF bipolar and horizontal cells, EPSCs rose and fell at significantly slower rates with time constants of $14.7 \pm 2.3 \mathrm{~ms}(n=10$; $p=0.009)$ and $120.3 \pm 34.4 \mathrm{~ms}(n=12 ; p=0.033)$, respectively. Significant rod/cone differences were also observed when comparisons were restricted to OFF bipolar cells [rod-driven $\tau_{\text {on }}=$ $16.5 \pm 2.8 \mathrm{~ms}(n=7)$ vs cone-driven $\tau_{\text {on }}=2.4 \pm 1.1 \mathrm{~ms}(n=4)$, $p=0.005$; rod-driven $\tau_{\text {decay }}=63.6 \pm 6.7 \mathrm{~ms}(n=9)$ vs conedriven $\left.\tau_{\text {decay }}=19.1 \pm 3.4 \mathrm{~ms}(n=6), p=0.0002\right]$.

The amount of charge transfer during the EPSC was used as a measure of glutamate release. As shown in Figure 2, increasing the duration of the depolarizing step caused an initial rapid rise in EPSC charge transfer followed by a slower increase with longer steps. The increase in charge transfer with step duration was fit with a biexponential function in both rod- and cone-driven cells. This two-phase rise in charge transfer is consistent with two kinetically distinct phases to release.

The most notable difference between rod- and cone-driven cells in Figure 2 is that the initial component of the charge transfer versus duration plot of cone-driven EPSCs was $>10$ times faster $\left(\tau_{1}=2.4 \mathrm{~ms}\right)$ than that of rod-driven EPSCs $\left(\tau_{1}=28 \mathrm{~ms}\right)$. In contrast to differences in the initial component of release, both rod- and cone-driven cells showed similar slow components $\left(\tau_{2}\right.$ $>500 \mathrm{~ms}$ ). Because these kinetic differences between rod- and cone-driven EPSCs were observed after broadening the EPSC with cyclothiazide, they are unlikely to reflect differences in glutamate receptor desensitization at rod- and cone-driven synapses. Instead, the more rapid initial component to the response/ duration plot and the more transient EPSCs in cone-driven neurons are more likely explained by an initial burst of release from cones that is $>10$ times faster than the burst of release from rods. Additional evidence for this is presented below.

Rod/cone differences in the EPSC waveform or kinetics of release do not reflect rod/cone differences in the activation or inactivation kinetics of $I_{\mathrm{Ca}}$. Consistent with Corey et al. (1984), we found that $I_{\mathrm{Ca}}$ activated rapidly (Fig. 3) with a biexponential time course, and the activation time constants did not differ significantly ( $p=0.18$ for comparison of $\tau_{1}$ values; $p=0.72$ for comparison of $\tau_{2}$ values $)$ between rods $\left(\tau_{1}=0.78 \pm 0.12 \mathrm{~ms} ; \tau_{2}=\right.$ $2.53 \pm 0.38 \mathrm{~ms})$ and cones $\left(\tau_{1}=0.97 \pm 0.06 \mathrm{~ms} ; \tau_{2}=2.37 \pm 0.25\right.$ $\mathrm{ms})$. Although $I_{\mathrm{Ca}}$ activates rapidly, it inactivates very slowly (Corey et al., 1984; Wilkinson and Barnes, 1996), with a time constant of $\sim 1.7 \mathrm{~s}$ in rods (Rabl and Thoreson, 2002). Despite the fact that $I_{\mathrm{Ca}}$ exhibited minimal inactivation during the first 100 ms of a depolarizing step in both rods and cones (Fig. 3), EPSCs decayed to near baseline during that same time period (Fig. 1). 


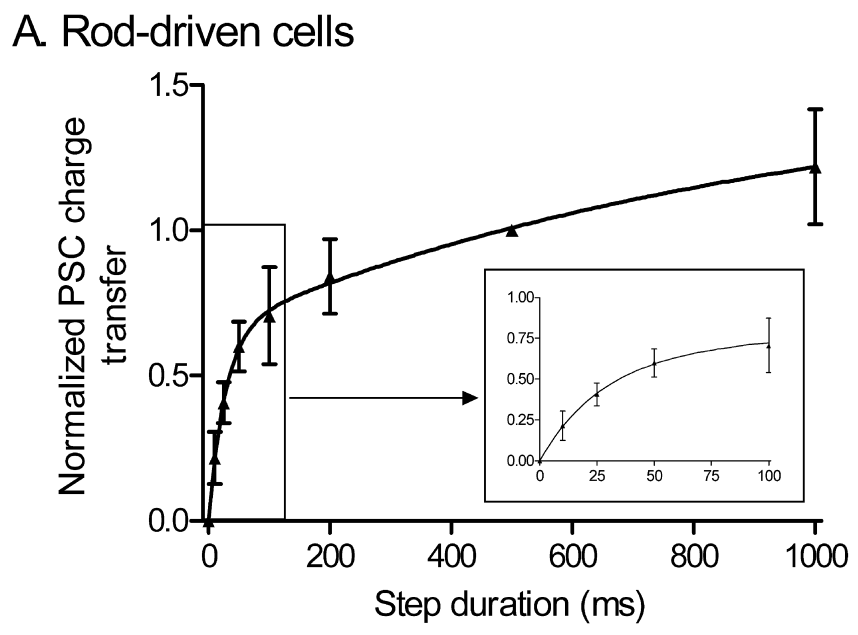

B. Cone-driven cells

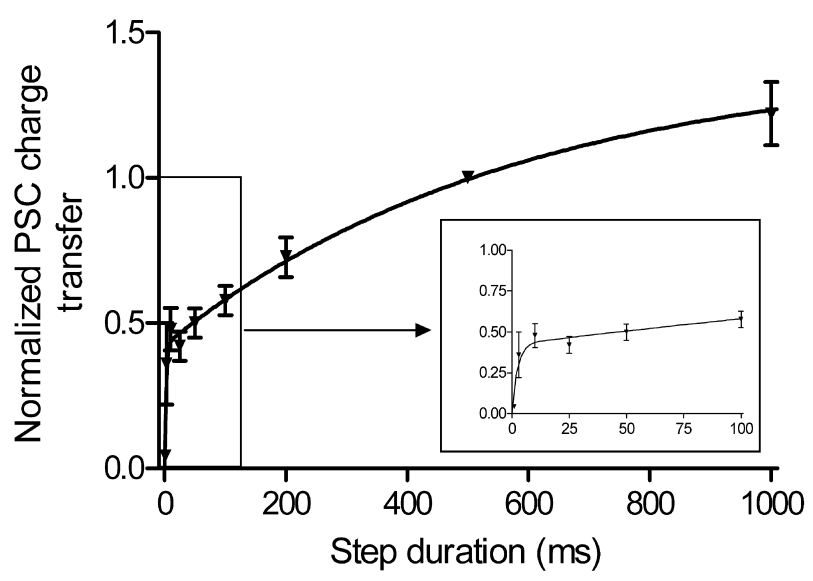

Figure 2. There are two kinetic components to EPSCs evoked by depolarizing steps applied to rods and cones. Increasing the duration (10 to $1000 \mathrm{~ms}$ ) of depolarizing steps $(-70$ to -10 $\mathrm{mV}$ ) applied to rods or cones caused a biexponential increase in the EPSC charge transfer measured in OFF bipolar and horizontal cells during paired recordings from retinal slices. Data from rod-driven cells are shown in $\boldsymbol{A}$, and data from cone-driven cells are shown in $\boldsymbol{B}$. Fits to shortduration steps are expanded in the insets. Postsynaptic recordings were obtained from both $0 \mathrm{FF}$ bipolar cells (rod driven, $n=5$; cone driven, $n=6$ ) and horizontal cells (rod driven, $n=3$; cone driven, $n=6)$ in the presence of cyclothiazide $(0.1 \mathrm{~mm})$. Data from six of the rod-driven cells were used for a similar comparison by Thoreson et al. (2004). No differences were detected between response/duration relationships in OFF bipolar and horizontal cells when synaptically stimulated by the same type of photoreceptor. Charge transfer during the EPSC was integrated and normalized to the charge transfer evoked by a $500 \mathrm{~ms}$ depolarizing step. Data were fit with dual-exponential functions (rods, $\tau_{1}=27.5 \mathrm{~ms}, \tau_{2}=954 \mathrm{~ms}$; cones, $\tau_{1}=2.4 \mathrm{~ms}, \tau_{2}=570$ ms). Error bars represent SEM.

This is consistent with findings of Singer and Diamond (2003) at the ribbon synapse from rod bipolar cells to AII amacrine cells showing that transient EPSCs in bipolar and horizontal cells can be evoked by sustained calcium entry into rod and cone terminals.

To test whether differences in EPSC kinetics arise from differences in rates of exocytosis at rod and cone terminals, we used capacitance recording techniques to measure release directly. Membrane capacitance was monitored by sinusoidally modulating $( \pm 15 \mathrm{mV})$ the membrane potential about a mean holding potential of $-70 \mathrm{mV}$ using a phase-lock amplifier integrated into the patch-clamp amplifier. Exocytosis was stimulated with depolarizing steps of variable duration (10-2000 ms) to -10 or -30 $\mathrm{mV}$. Capacitance measurement was suspended during the test step. As shown in Figure 3, inward $I_{\mathrm{Ca}}$ stimulated by a depolariz-

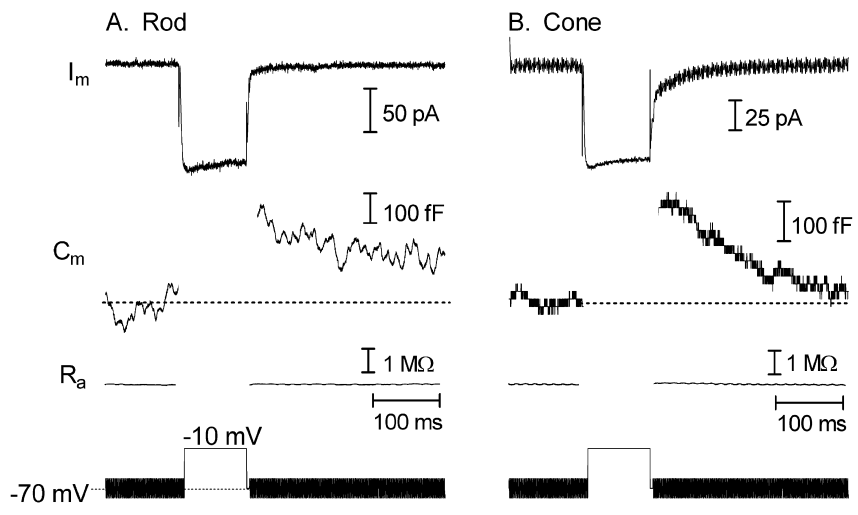

Figure 3. Membrane depolarization triggered exocytosis from rods and cones in a retinal slice. Traces show membrane current $\left(I_{\mathrm{m}}\right)$, membrane capacitance $\left(C_{\mathrm{m}}\right)$, access resistance $\left(R_{\mathrm{a}}\right)$, and the stimulus protocol. Capacitance was monitored in a $\operatorname{rod}(\boldsymbol{A})$ and a cone $(\boldsymbol{B})$ with a phase-lock amplifier while sinusoidally varying the holding potential $(600 \mathrm{~Hz}, \pm 15 \mathrm{mV})$ about the mean holding potential of $-70 \mathrm{mV}$. Phase tracking was gated out during the depolarizing test step to $-10 \mathrm{mV}$ ( $100 \mathrm{~ms}$ ) and for $3 \mathrm{~ms}$ after the step. Passive membrane resistances of 500 $\mathrm{M} \Omega$ in the rod and $800 \mathrm{M} \Omega$ in the cone were leak subtracted from the current traces.

ing step (200 ms) evoked exocytotic increases in membrane capacitance in both rod and cone cells. When capacitance monitoring was resumed after the test step, the membrane capacitance had increased by $240 \mathrm{fF}$ in the rod and $300 \mathrm{fF}$ in the cone. Assuming a single vesicle capacitance of $57 \mathrm{aF}$ (Thoreson et al., 2004), these capacitance increases reflect the fusion of 4210 and 5260 vesicles, respectively. The membrane capacitance typically began to recover within a few hundred milliseconds after the test step. The time required for $50 \%$ recovery of the capacitance after a 500 ms step test step averaged $350 \mathrm{~ms}$ in cones and $>500 \mathrm{~ms}$ in rods. (A more precise estimate is not available for rods, because a few cells did not achieve 50\% recovery before the end of the $1.5 \mathrm{~s}$ trial.)

A number of different observations indicate that observed capacitance changes reflected true changes in membrane capacitance. Depolarization-evoked capacitance increases were not a result of changes in access resistance, because it remained unchanged in these experiments (Figs. 3, 4). Sufficiently large changes in membrane resistance can also produce artifactual changes in capacitance (Joshi and Fernandez, 1988; Gillis, 1995). To minimize conductance changes, we inhibited tail currents arising from activation of calcium-activated chloride currents by applying niflumic acid $(0.2 \mathrm{~mm})$. Although niflumic acid blocked much of this current, residual tail currents were sometimes observed with longer steps. With $2 \mathrm{~s}$ steps, tail currents in rods averaged $79 \pm 12 \mathrm{pA}(n=12)$, whereas those in cones averaged $20 \pm 7 \mathrm{pA}(n=10)$. Nearly complete block of tail currents in cones is consistent with the efficacy of niflumic acid in cones shown by Barnes and Deschenes (1992). The time constant for tail current inactivation increased as a function of duration; the increase in inactivation time constants exhibited a time constant of $1.05 \mathrm{~s}$ in rods and $0.48 \mathrm{~s}$ in cones, indicating that residual tail currents were often still present at the time that capacitance measurements were made. However, the presence of residual tail currents did not appear to significantly affect capacitance measurements. Depolarization-evoked capacitance changes were observed in cells exhibiting little or no tail current (Fig. 3), and conversely, in control experiments omitting niflumic acid from the bathing medium, tail currents could be evoked without accompanying capacitance changes (Fig. 4). Furthermore, the time course of capacitance changes did not mirror that of the tail cur- 
A

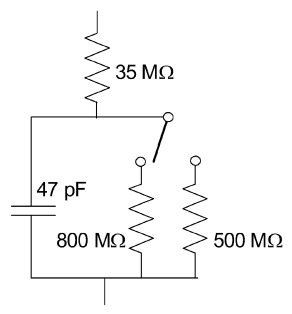

B
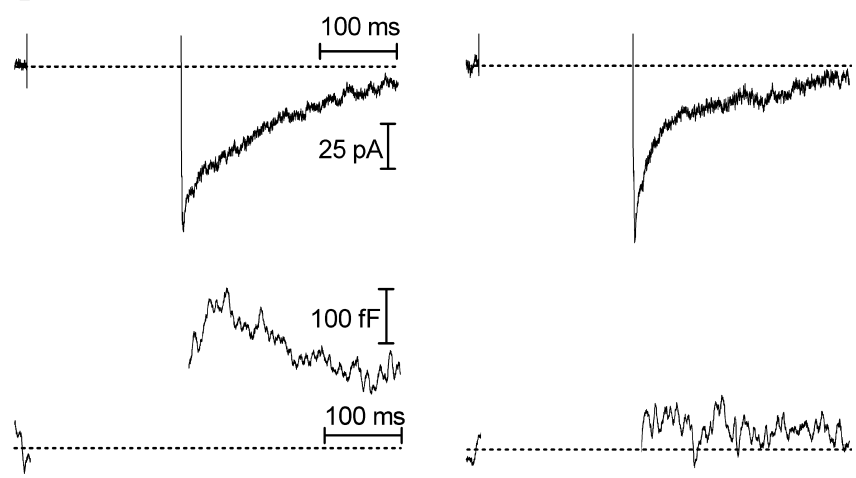

Figure 4. Tail currents could be evoked without altering capacitance measurements. $\boldsymbol{A}$, Little or no capacitance change was observed when the membrane resistance in a model cone cell was manually reduced from 800 to $500 \mathrm{M} \Omega$ to simulate the large conductance change accompanying a tail current. Capacitance was monitored using the phase-tracking feature of the Optopatch amplifer as described in Materials and Methods. $\boldsymbol{B}$, An example from a rod photoreceptor showing that tail currents could be evoked in cells without accompanying capacitance changes. In this experiment, niflumic acid was omitted to permit the generation of tail currents. Early in the recording from this rod, the depolarizing test step ( $200 \mathrm{~ms},-70$ to -10 $\mathrm{mV}$ ) evoked an inward tail current at the end of the step (top left) and a large capacitance increase (bottom left). Application of the same test step 15 min later evoked similar inward tail currents (top right) but no capacitance increase (bottom right).

rent (Fig. 4). Using a different approach, we simulated the effects of a large tail current that might be observed in the absence of niflumic acid using a model circuit that mimicked the passive membrane properties of a cone. Reducing the membrane resistance in this circuit from 800 to $500 \mathrm{M} \Omega$ produced little or no capacitance change (Fig. 4). Finally, as described below (see Figs. $6,7)$, there was close agreement between capacitance increases and EPSC charge transfer measurements providing additional validation of capacitance increases as measures for exocytosis.

Increasing the duration of the depolarizing step increased the amplitude of the evoked increase in membrane capacitance. Figure $5 \mathrm{~A}$ shows the capacitance increases evoked in a rod by depolarizing steps to $-10 \mathrm{mV}$ of $10 \mathrm{~ms}(30 \mathrm{fF}), 100 \mathrm{~ms}(80 \mathrm{fF})$, and $1000 \mathrm{~ms}(110 \mathrm{fF})$. Figure $5 B$ shows the capacitance increases evoked in a cone by depolarizing steps of $10 \mathrm{~ms}$ ( $170 \mathrm{fF}), 100 \mathrm{~ms}$ $(430 \mathrm{fF})$, and $1000 \mathrm{~ms}$ (570 fF). Figure 6 plots capacitance increases as a function of step duration from the entire sample of rods and cones. Consistent with the results from paired recordings (Fig. 2) as well as previous results from isolated rods (Thoreson et al., 2004) and other ribbon synapses (Parsons et al., 1994; von Gersdorff and Matthews, 1994a), capacitance/duration relationships in rods and cones were fit by dual exponential functions, suggesting there are two kinetically distinct phases to exocytosis. The initial fast component is thought to reflect rapid release of a pool of vesicles that are primed for release and the slow component continuing tonic release of vesicles (Lenzi and von Gersdorff, 2001).

As found in EPSC experiments, the initial fast component of release was $>10$-fold faster in cones $\left(\tau_{1}=3 \mathrm{~ms}\right)$ than $\operatorname{rods}\left(\tau_{1}=\right.$
$46 \mathrm{~ms}$ ). The amplitude of the fast component in cones (306 fF) corresponds to release of 5370 vesicles. This was approximately twice as large as the fast component in rods ( $132 \mathrm{fF})$, which corresponds to release of 2300 vesicles or slightly less than one-half of the vesicles tethered to the ribbons in a presynaptic terminal (4970 vesicles) (Thoreson et al., 2004). The initial time constant obtained from EPSC measurements of release was faster than that found using capacitance data. This difference is a result of integrating charge transfer over the entire EPSC and thus counting vesicles for a slightly longer time window than in capacitance experiments. By restricting the integration period to the duration of the test step, the initial time constant closely approached values found in capacitance experiments.

In both rods and cones, there was also a slow sustained component to release with time constants exceeding $1 \mathrm{~s}$. Over time, this slow component contributed a substantial amount of release: by the end of the $2 \mathrm{~s}$ test step, approximately twice as many vesicles had been released than were released by the initial fast component (rods, $298 \pm 58 \mathrm{fF}$ or 5228 vesicles; cones, $656 \pm 53 \mathrm{fF}$ or 11,500 vesicles). This should be viewed as a lower-bound estimate of the total number of vesicles that were released, because endocytosis may have reduced the amplitude of capacitance responses evoked by longer steps ( $\geq 500 \mathrm{~ms}$ ).

We examined whether rod/cone kinetic differences persisted when cells were stimulated with steps from -70 to $-30 \mathrm{mV}$, close to the physiological voltage range. As with steps to $-10 \mathrm{mV}$ shown in Figure 6, there were two kinetic components to the capacitance increase as a function of step duration. Capacitance increases were well fit with biexponential functions using time constants of 20 and $640 \mathrm{~ms}$ in rods ( $n=6-13$ for every point) and 3 and $230 \mathrm{~ms}$ in cones $(n=8-13$ for every point; data not shown). Although the best-fit initial component for release from rods evoked by steps to $-30 \mathrm{mV}$ was somewhat faster than that obtained with steps to $-10 \mathrm{mV}$, the difference was not significant and remained nearly 10 -fold faster than the initial component in cones. This suggests that significant kinetic differences in release from rods and cones are likely to persist in the physiological voltage range.

To test the potential contribution of rod-rod coupling in the amphibian retinal slice to the measured kinetics of release from rods, we repeated capacitance/duration (10-1000 ms) measurements on enzymatically isolated rods with intact synaptic terminals. The capacitance increased with a biexponential time course in isolated rods that was not significantly different from that found in rods from the slice $\left(\mathrm{t}_{1}=87 \mathrm{~ms}, \mathrm{t}_{2}=4.5 \mathrm{~s}, n=9\right.$; comparison of both components, $p=0.78$; comparison of $\tau_{1}$ values alone, $p=0.49$ ).

In a previous study, we found a biexponential rise in release from isolated rods as a function of depolarizing step duration, but the initial component of release was much faster than that found in the present study $\left(\tau_{1}=6 \mathrm{~ms}\right)$ (Thoreson et al., 2004). The data described above suggest that the difference was not a result of the use of isolated cells. Differences are also not because of use of a software-based dual sine wave protocol for the measurement of capacitance, because we repeated measurements on rods in the slice using the dual sine wave technique (jClamp; Scisoft) and obtained time constants for release that were not significantly different from those found with the Optopatch phase tracking circuitry $\left(\tau_{1}=100 \mathrm{~ms}, \tau_{2}>10 \mathrm{~s}, n=6-8\right.$ per data point; comparison of both components, $p=0.72$; comparison of $\tau_{1}$ values alone, $p=0.76$ ). Differences instead appear to be attributable to the fact that in the previous study, capacitance changes were measured during the $10 \mathrm{~ms}$ immediately after the step, whereas in the 
current study, capacitance changes were measured 25-50 ms after the step. When data from Thoreson et al. (2004) were reanalyzed by measuring of capacitance changes 25-50 ms after the step, we obtained capacitance values $\sim 40 \mathrm{fF}$ smaller than those obtained previously, and these values yielded a slower initial time constant in good agreement with that of the present study $\left(\tau_{1}=21 \mathrm{~ms} ; \tau_{2}=436 \mathrm{~s}\right)$.

To better compare capacitance and EPSC measures of exocytosis, we plotted the EPSC charge transfer against the capacitance change evoked by steps of the same duration (Fig. 7). Charge transfer values were normalized to the charge transfer evoked by a $500 \mathrm{~ms}$ step. The two measures of release were strongly correlated in both rods $\left(r^{2}=0.95\right)$ and cones $\left(r^{2}=0.91\right)$. Increases in capacitance and PSC charge transfer evoked by steps to $-30 \mathrm{mV}$ in rods were also closely correlated $\left(r^{2}=0.87\right.$; data not shown). The close correlation between these convergent measures of exocytosis substantiates the finding that there are significant differences in the kinetics of release from rod and cone terminals.

\section{Discussion}

The results of this study show that there are two kinetically distinct phases to vesicular glutamate release from synaptic terminals of rods and cones and that the initial phase of release proceeds much more rapidly in cones than rods. Almost identical results were obtained using two convergent techniques for measuring release. In paired whole-cell recording experiments in which the depolarization-evoked EPSC in OFF bipolar and horizontal cells was used as an index for release, the initial component of release from cones was found to be $>10$-fold faster than the initial component of release from rods. Likewise, membrane capacitance increases that provide a more direct measure of exocytosis showed that the fast component of release from cones increased with a time constant of $3 \mathrm{~ms}$, whereas the fast component of release from rods increased with a time constant of $46 \mathrm{~ms}$. The fast initial component of release is thought to represent release of a pool of synaptic vesicles that is primed for release (Mennerick and Matthews, 1996; von Gersdorff et al., 1996; Moser and Beutner, 2000; Thoreson et al., 2004). The finding that cones exhibit a faster initial kinetic component to release indicates that vesicles in this readily releasable pool are released more rapidly from cones than the analogous pool of vesicles in rods.

In a previous study on isolated rods, Thoreson et al. (2004) also found a biexponential rise in release as a function of step duration. However, the time constant for the initial component of release $\left(\tau_{1}=6 \mathrm{~ms}\right)$ was much faster than that found in the present study. Our results indicated that the more rapid kinetics found previously cannot be explained by an absence of coupling in isolated rods or the use of dual sine wave capacitance measurement techniques. However, reanalyzing the data of Thoreson et al. (2004) by measuring capacitance increases 25-50 ms after the step, rather than during the first $10 \mathrm{~ms}$ after the step as done previously, we obtained smaller capacitance increases resulting in a slower initial time constant for release $\left(\tau_{1}=21 \mathrm{~ms}\right)$, in good agreement with the present data. The observation that capaci- tance changes measured during the first $10 \mathrm{~ms}$ after the step were $\sim 40 \mathrm{fF}$ larger than capacitance changes measured 25-50 ms after the step suggests there was a brief capacitance increase that subsided within $25 \mathrm{~ms}$. Such a brief capacitance increase could conceivably be produced by kiss-and-run exocytosis, but the finding that the rise in the PSC as a function of duration closely paralleled the rise in capacitance measured $25-50 \mathrm{~ms}$ after the step suggests that the additional capacitance increase seen during the first 10 $\mathrm{ms}$ had a negligible postsynaptic effect and is therefore probably unrelated to synaptic transmission (e.g., reflecting extrasynaptic exocytosis or a nonvesicular artifact).

Concurrent endocytosis can produce net reductions in exocytotic capacitance increases. The time constant for endocytosis in rods has been estimated at $\sim 700 \mathrm{~ms}$ (Rieke and Schwartz, 1996), consistent with our finding that $50 \%$ recovery required $>500 \mathrm{~ms}$. There was a good linear correlation between the amplitude of depolarization-evoked capacitance increases and charge transfer during EPSCs that intercepts the origin in rods (Fig. 7), suggesting that endocytosis is minimal during test steps up to $1 \mathrm{~s}$ in duration, perhaps because endocytosis may be inhibited during the test step by elevated $\mathrm{Ca}^{2+}$ levels, as found in retinal bipolar cells (von Gersdorff and Matthews, 1994b). Membrane endocytosis was somewhat more rapid in cones achieving 50\% recovery after $\sim 350 \mathrm{~ms}$ and may have therefore have reduced the size of capacitance increase evoked by steps $\geq 500 \mathrm{~ms}$ in cones (Fig. 6). A reduction of capacitance responses with long steps would explain the observation that the linear correlation between capacitance and PSC measures of release in cones, unlike rods, intercepted the ordinate above $0 \mathrm{fF}$ (Fig. 7). Although endocytosis may have reduced the amplitude of capacitance increases evoked by long depolarizing steps, particularly in cones, endocytosis did not appear to significantly impact measurements made during the first few hundred milliseconds and was thus unlikely to influence determination of the kinetics of the initial fast component of release. Furthermore, similar release kinetics in rod and cone terminals was found using PSC measurements of release that are insensitive to endocytosis.

Although an exhaustive determination of the mechanisms re- 

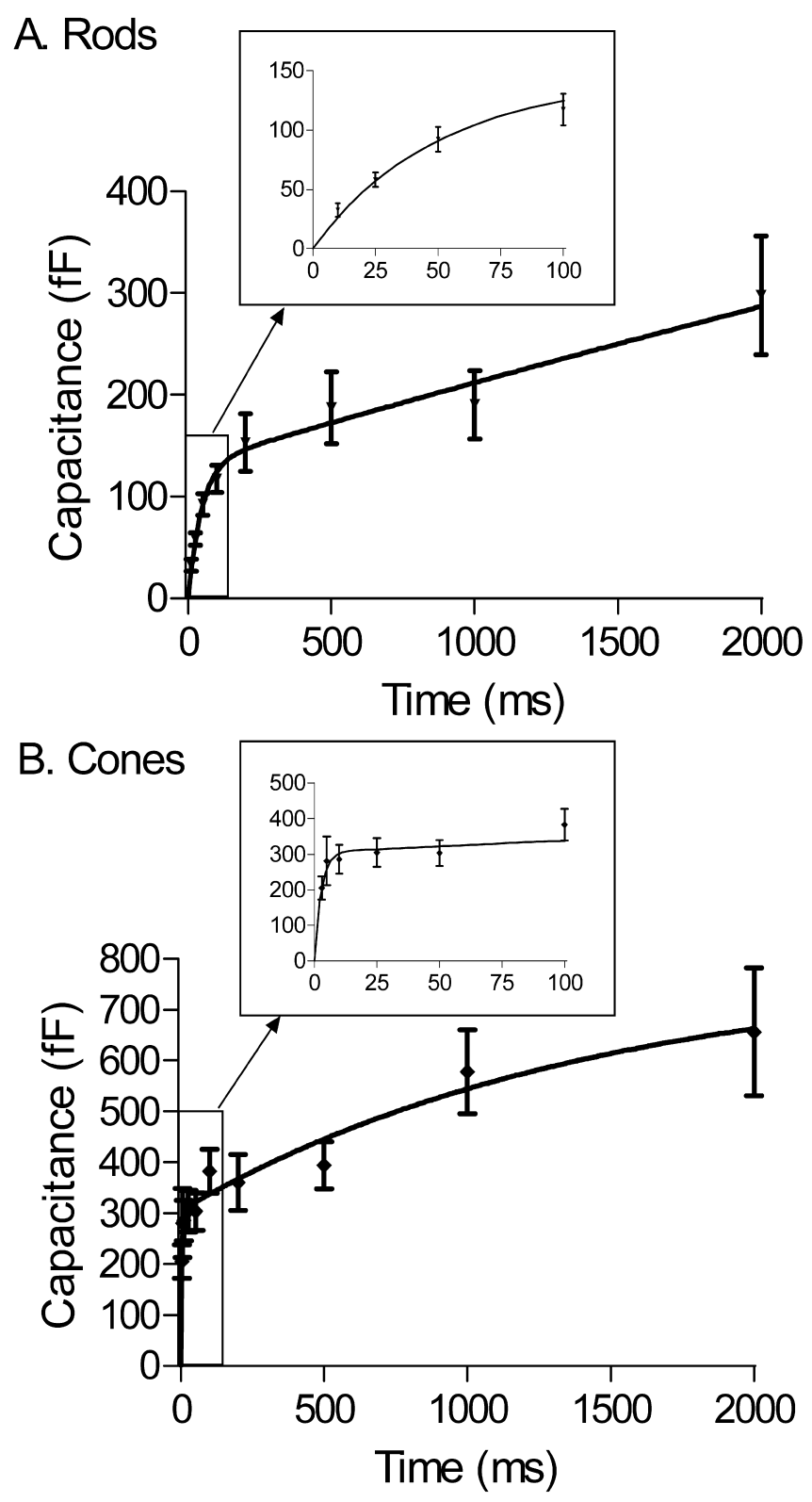

Figure 6. There are two kinetic components to exocytosis from rods $(\boldsymbol{A})$ and cones $(\boldsymbol{B})$. Depolarization-evoked capacitance increases were plotted as a function of step duration. Capacitance/duration relationships were fit with dual-exponential functions. Fits to shortduration steps are expanded in the insets. Rods, $\tau_{1}=46 \mathrm{~ms}$, Amplitude ${ }_{1}\left(\mathrm{~A}_{1}\right)=132 \mathrm{fF}, \tau_{2}=$ $14 \mathrm{~s}$, Amplitude A $_{2}\left(\mathrm{~A}_{2}\right)=1170 \mathrm{fF}$; cones, $\tau_{1}=2.7 \mathrm{~ms}, \mathrm{~A}_{1}=306 \mathrm{fF}, \tau_{2}=1.4 \mathrm{~s}, \mathrm{~A}_{2}=474 \mathrm{fF}$. Sample sizes: rods, $10 \mathrm{~ms}, n=9 ; 25 \mathrm{~ms}, n=11 ; 50 \mathrm{~ms}, n=10 ; 100 \mathrm{~ms}, n=12 ; 200 \mathrm{~ms}, n=$ $11 ; 500 \mathrm{~ms}, n=10 ; 1000 \mathrm{~ms}, n=10 ; 2000 \mathrm{~ms}, n=7 ;$ cones, $3 \mathrm{~ms}, n=9 ; 5 \mathrm{~ms}, n=5 ; 10 \mathrm{~ms}$, $n=16 ; 25 \mathrm{~ms}, n=14 ; 50 \mathrm{~ms}, n=13 ; 100 \mathrm{~ms}, n=20 ; 200 \mathrm{~ms}, n=12 ; 500 \mathrm{~ms}, n=11 ; 1000$ $\mathrm{ms}, n=11 ; 2000 \mathrm{~ms}, n=12$. Error bars represent SEM.

sponsible for faster release kinetics in cones compared with rods is beyond the scope of the present study, we can eliminate a number of possibilities: (1) The faster release kinetics measured in cones is unlikely to be artifacts arising from differences in passive membrane properties, because the membrane time constants for cones were slower, not faster, than those in rods $(2.8 \mathrm{vs}$ $2.1 \mathrm{~ms})$. (2) The finding that the kinetics of release from isolated rods was similar to that in rods from the retinal slice preparation indicates that the slow kinetics of release from rods is also not a consequence of extensive rod-rod coupling in the amphibian retina. (3) The kinetics of release at bipolar cell terminals has been

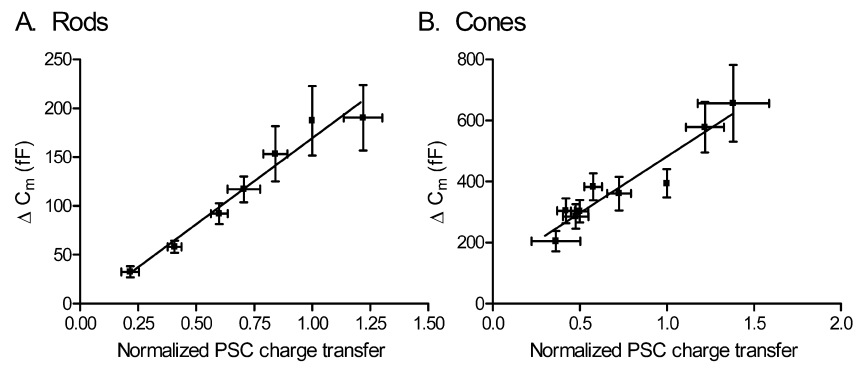

Figure 7. Capacitance $\left(C_{\mathrm{m}}\right)$ changes evoked in rods $(\boldsymbol{A})$ or cones $(\boldsymbol{B})$ by depolarizing steps $(-70$ to $-10 \mathrm{mV})$ of varying duration (3-1000 ms in rods, 3-2000 ms in cones) are linearly correlated with the integrated charge transfer of EPSCs in OFF bipolar and horizontal cells (rods, $r^{2}=0.95$; cones, $\left.r^{2}=0.91\right)$. Error bars represent SEM.

shown to be limited by the activation kinetics of $I_{\mathrm{Ca}}$ (Mennerick and Matthews, 1996). The observation that the activation rate of $I_{\mathrm{Ca}}\left(\tau_{1}, \sim 1 \mathrm{~ms} ; \tau_{2}, \sim 2.5 \mathrm{~ms}\right)$ is similar to activation of the initial component of exocytosis in cones $\left(\tau_{1}, \sim 3 \mathrm{~ms}\right)$ is consistent with the possibility that $I_{\mathrm{Ca}}$ activation rates may limit release kinetics at cone terminals. However, the $46 \mathrm{~ms}$ time constant for activation of the initial component of exocytosis in rods is substantially slower than the rate of $I_{\mathrm{Ca}}$ activation, suggesting that other factors limit the kinetics of release from rods. (4) $I_{\mathrm{Ca}}$ inactivates with similar slow kinetics in both rods and cones, indicating that transient EPSCs do not reflect rapid inactivation of $I_{\mathrm{Ca}}$ (Fig. 3) (Corey et al., 1984; Rabl and Thoreson, 2002). Other remaining possibilities that might explain kinetic differences between rods and cones include differences in properties of exocytotic proteins (Wang et al., 2003), diffusional distance between calcium channels and release sites, and intracellular calcium handling in rod and cone terminals (Krizaj et al., 2003).

Release kinetics has been shown to shape postsynaptic responses (Diamond and Jahr, 1995; Isaacson and Walmsley, 1995), and the rapid release of vesicles from cones may likewise contribute to the faster initial rise of EPSCs in cone-driven cells compared with rod-driven cells (Fig. 1). Similar to the rod bipolar ribbon synapse onto AII amacrine cells (Singer and Diamond, 2003), the EPSC decayed to near baseline despite the continued influx of calcium presynaptically and the block of postsynaptic AMPA receptor desensitization by cyclothiazide. The decline in the EPSC is thus more readily explained by depletion of the pool of releasable vesicles. Furthermore, because cones initially release their vesicles more rapidly than rods, the readily releasable pool of vesicles should also be depleted more rapidly in cones than rods. The more rapid depletion of vesicles from cone terminals can explain the more rapid decline in the EPSC observed in conedriven cells compared with rod-driven cells (Fig. 1). Differences in the properties of glutamate receptors can also contribute to shaping EPSC kinetics (DeVries, 2000), although recent studies from our laboratory reveal no major pharmacological differences in the glutamate receptors contacted by rods and cones (our unpublished observations).

The influence of release kinetics on the shape of PSCs evoked by depolarizing steps from $-70 \mathrm{mV}$ is relatively straightforward compared with their effects on light-evoked responses. Lightevoked changes in synaptic output combine the replenishment of vesicles and decreased exocytosis that accompany hyperpolarization at light onset with the increased rates of exocytosis evoked by depolarization at light offset. A complete analysis of the impact of these various factors on the kinetic differences of light-evoked responses has not yet been performed, but the present results 
showing rod/cone differences in the initial fast component using test steps near the dark potential suggest that differences in the rates of exocytosis may influence synaptic output in response to light-evoked changes in membrane potential. Because of the rapid depletion of vesicles that necessarily accompanies rapid release, it is unlikely that the extremely fast release from cones can be maintained for very long. Thus, when the photoreceptor is maintained at depolarized potentials under physiological conditions, slow sustained components of release are likely to become increasingly important. However, the underlying capability of cones to release vesicles rapidly may allow a brief but substantial increase in exocytosis when cones depolarize in response to a reduction in light intensity. A transient increase in synaptic output would contribute to the faster synaptic impulse response of cones compared with rods (Schnapf and Copenhagen, 1982; Copenhagen et al., 1983).

\section{References}

Barnes S, Deschenes MC (1992) Contribution of Ca and Ca-activated Cl channels to regenerative depolarization and membrane bistability of cone photoreceptors. J Neurophysiol 68:745-755.

Baylor DA, Fettiplace R (1977) Transmission from photoreceptors to ganglion cells in turtle retina. J Physiol (Lond) 271:391-448.

Baylor DA, Hodgkin AL (1973) Detection and resolution of visual stimuli by turtle photoreceptors. J Physiol (Lond) 234:163-198.

Copenhagen DR, Ashmore JF, Schnapf JK (1983) Kinetics of synaptic transmission from photoreceptors to horizontal and bipolar cells in turtle retina. Vision Res 23:363-369.

Corey DP, Dubinsky JM, Schwartz EA (1984) The calcium current in inner segments of rods from the salamander (Ambystoma tigrinum) retina. J Physiol (Lond) 354:557-575.

DeVries SH (2000) Bipolar cells use kainate and AMPA receptors to filter visual information into separate channels. Neuron 28:847-856.

DeVries SH (2001) Exocytosed protons feedback to suppress the $\mathrm{Ca}^{2+}$ current in mammalian cone photoreceptors. Neuron 32:1107-1117.

Diamond JS, Jahr CE (1995) Asynchronous release of synaptic vesicles determines the time course of the AMPA receptor-mediated EPSC. Neuron 15:1097-1107.

Gillis KD (1995) Techniques for membrane capacitance measurements. In: Single channel recording, Ed 2 (Sakmann B, Neher E, eds), pp 155-198. New York: Plenum.

Isaacson JS, Walmsley B (1995) Counting quanta: direct measurements of transmitter release at a central synapse. Neuron 15:875-884.

Johnson SL, Thomas MV, Kros CJ (2002) Membrane capacitance measurement using patch clamp with integrated self-balancing lock-in amplifier. Pflügers Arch 443:653-663.

Joshi C, Fernandez JM (1988) Capacitance measurements: an analysis of the phase detector technique used to study exocytosis and endocytosis. Biophys J 53:885-892.

Korenbrot JI, Rebrik TI (2002) Tuning outer segment $\mathrm{Ca}^{2+}$ homeostasis to phototransduction in rods and cones. Adv Exp Med Biol 514:179-203.

Krizaj D, Lai FA, Copenhagen DR (2003) Ryanodine stores and calcium regulation in the inner segments of salamander rods and cones. J Physiol (Lond) 547:761-774.

Lasater EM, Normann RA, Kolb H (1989) Signal integration at the pedicle of turtle cone photoreceptors: an anatomical and electrophysiological study. Vis Neurosci 2:553-564.

Lenzi D, von Gersdorff H (2001) Structure suggests function: the case for synaptic ribbons as exocytotic nanomachines. BioEssays 23:831-840.
Maple BR, Gao F, Wu SM (1999) Glutamate receptors differ in rod- and cone-dominated off-center bipolar cells. NeuroReport 10:3605-3610.

Mennerick S, Matthews G (1996) Ultrafast exocytosis elicited by calcium current in synaptic terminals of retinal bipolar neurons. Neuron 17:1241-1249.

Moser T, Beutner D (2000) Kinetics of exocytosis and endocytosis at the cochlear inner hair cell afferent synapse of the mouse. Proc Natl Acad Sci USA 97:883-888.

Parsons TD, Lenzi D, Almers W, Roberts WM (1994) Calcium-triggered exocytosis and endocytosis in an isolated presynaptic cell: capacitance measurements in saccular hair cells. Neuron 13:875-883.

Pasino E, Marchiafava PL (1976) Transfer properties of rod and cone cells in the retina of the tiger salamander. Vision Res 16:381-386.

Rabl K, Thoreson WB (2002) Calcium-dependent inactivation and depletion of synaptic cleft calcium ions combine to regulate rod calcium currents under physiological conditions. Eur J Neurosci 16:2070-2077.

Rieke F, Schwartz E (1996) Asynchronous transmitter release: control of exocytosis and endocytosis at the salamander rod synapse. J Physiol (Lond) 493:1-8.

Schmitz Y, Witkovsky P (1997) Dependence of photoreceptor glutamate release on a dihydropyridine-sensitive calcium channel. Neuroscience 78:1209-1216.

Schnapf JL, Copenhagen DR (1982) Differences in the kinetics of rod and cone synaptic transmission. Nature 296:862-864.

Sherry DM, Bui DD, Degrip WJ (1998) Identification and distribution of photoreceptor subtypes in the neotenic tiger salamander retina. Vis Neurosci 15:1175-1187.

Singer JH, Diamond JS (2003) Sustained $\mathrm{Ca}^{2+}$ entry elicits transient postsynaptic currents at a retinal ribbon synapse. J Neurosci 23:10923-10933.

Stella Jr SL, Thoreson WB (2000) Differential modulation of rod and cone calcium currents in tiger salamander retina by D2 dopamine receptors and cAMP. Eur J Neurosci 12:3537-3548.

Taylor WR, Morgans C (1998) Localization and properties of voltage-gated calcium channels in cone photoreceptors of Tupaia belangeri. Vis Neurosci 15:541-552.

Thoreson WB, Miller RF (1996) Removal of extracellular chloride suppresses transmitter release from photoreceptor terminals in the mudpuppy retina. J Gen Physiol 107:631-642.

Thoreson WB, Nitzan R, Miller RF (1997) Reducing extracellular $\mathrm{Cl}^{-}$suppresses dihydropyridine-sensitive $\mathrm{Ca}^{2+}$ currents and synaptic transmission in amphibian photoreceptors. J Neurophysiol 77:2175-2190.

Thoreson WB, Bryson EJ, Rabl K (2003) Reciprocal interactions between calcium and chloride in rod photoreceptors. J Neurophysiol 90:1747-1753.

Thoreson WB, Rabl K, Townes-Anderson E, Heidelberger R (2004) A highly $\mathrm{Ca}^{2+}$-sensitive pool of vesicles contributes to linearity at the rod photoreceptor ribbon synapse. Neuron 42:595-605.

von Gersdorff H, Matthews G (1994a) Dynamics of synaptic vesicle fusion and membrane retrieval in synaptic terminals. Nature 367:735-739.

von Gersdorff H, Matthews G (1994b) Inhibition of endocytosis by elevated internal calcium in a synaptic terminal. Nature 370:652-655.

von Gersdorff H, Vardi E, Matthews G, Sterling P (1996) Evidence that vesicles on the synaptic ribbon of retinal bipolar neurons can be rapidly released. Neuron 16:1221-1227.

Wang CT, Lu JC, Bai J, Chang PY, Martin TF, Chapman ER, Jackson MB (2003) Different domains of synaptotagmin control the choice between kiss-and-run and full fusion. Nature 424:943-947.

Wilkinson MF, Barnes S (1996) The dihydropyridine-sensitive calcium channel subtype in cone photoreceptors. J Gen Physiol 107:621-630.

Witkovsky P, Stone S (1983) Rod and cone inputs to bipolar and horizontal cells of the Xenopus retina. Vision Res 23:1251-1258. 